\title{
Perineal nodular swelling in a recreational cyclist: diagnosis and discussion
}

\author{
Stefaan Van de Perre • Filip M. Vanhoenacker • \\ Luc Vanstraelen • Jan Gaens • Maarten Michiels
}

Published online: 24 June 2009

(C) ISS 2009

\section{Diagnosis}

The diagnosis of a biker's nodule was made, based merely on the clinical history, the typical location and the imaging findings.

\section{Discussion}

A biker's nodule is a lesser known condition of the perineum that affects cyclists. The lesion has been previously known by many synonyms, including 'third or supernumerary testicle', 'accessory testicle', 'perineal hygroma or induration' [1-5]. It is typically located in the paramedian region of the perineum, posterior to the scrotal sac, and is covered with normal skin. The size of the lesion rarely exceeds $3 \mathrm{~cm}$ [1-5].

Symptoms include pain on pressure and when sitting on the saddle, which often forces the athlete to give up riding the bicycle. On palpation, the nodule is solid and

The case presentation can be found at doi: 10.1007/s00256-009-0730-x

S. Van de Perre $\cdot$ L. Vanstraelen

Department of Radiology, Heilig Hartziekenhuis,

Mol, Belgium

F. M. Vanhoenacker $(\square)$

Department of Radiology, University Hospital Antwerp,

Wilrijkstraat 10 ,

2650 Edegem, Belgium

e-mail: filip.vanhoenacker@telenet.be

J. Gaens

Department of Radiology, A.Z. St. Dimpna,

Geel, Belgium

M. Michiels

Department of Surgery, Heilig Hartziekenhuis,

Mol, Belgium sometimes adherent to the adjacent soft tissue $[2,5]$. The lesion is believed to result from increased pressure, vibration and friction between the ischial tuberosities and the hard saddle, characteristic of racing cycles or mountain bikes, with constant rubbing of the superficial perineal fascia against the bony structures [1-5].

Histopathology shows a myxoid degeneration of the fatty tissue and collagen fibers overlying the ischial tuberosities, caused by necrosis of the superficial perineal fascia, sometimes with formation of pseudocysts [1-5]. The lesion is not well vascularized [2].

Imaging characteristics are only rarely reported. Ultrasound shows a hypo-echoic nodule, with absence of any increased power Doppler signal [1]). Small internal cystic areas may be seen as well [1]. On computed tomography (CT), the lesion shows no uptake of contrast agent [3], which is explained by the hypovascular nature on histology. To our knowledge, magnetic resonance imaging (MRI) has not been reported. As in CT, there is no uptake of contrast medium. The primary role of imaging is to determine the exact extent of the lesion. Ultrasound and MRI are particularly helpful in locating the lesion in the subcutaneous fatty tissue and close to the ischial tuberosities. In most clinical scenarios, ultrasound will suffice for imaging evaluation. Imaging has an additional role in the differentiation between 'biker's nodule' and other causes of a perineal swelling. The differential diagnosis [1, 3, 5] includes abscess, epidermal cyst, (cutaneous) adnexial mass, lipoma, and malignant tumor (soft tissue sarcoma or metastasis).

In biker's nodules, signs of inflammation or abscess formation are lacking, and the absence of contrast agent enhancement on MRI excludes malignancy.

The primary therapy consists of avoidance of the causative factor. Rest alone is generally not sufficient as therapy. The condition almost systematically imposes saddle adjustments 
or change (different saddle shape and coverings in order to improve individual saddle fitting). Special attention should also be given to the pants of the cyclist. If the lesion is therapy resistant, some authors recommend local injection of corticosteroids or hyaluronidase. If none of these therapies is efficacious, surgery can be considered [1-5].

A high index of suspicion is required for diagnosis, and, therefore, clinical history is the most important clue to the correct diagnosis.

When a soft tissue nodule in the perineal region is seen in a cyclist, both the clinician and the radiologist should consider the diagnosis of a 'biker's nodule'.

\section{References}

1. Köhler P, Utermann S, Kahle B, Hartschuh W. 'Biker's nodule'die perineale knotige Induration des Radsportlers. Hautarzt. 2000;51:763-5.

2. Bauer P, Etienney I. Affections périnéales liées au sport. Gastroenterol Clin Biol. 2003;27:999-1007.

3. Rodier JF, Janser JC, Rodier D. Induration nodulaire périnéale. J Chir (Paris). 1992;129:49-50.

4. de Saint Aubain Somerhausen N, Geurde B, Couvreur Y. Perineal nodular induration: the 'third testicle of the cyclist', an underrecognized pseudotumour. Histopathology. 2003;42:615-22.

5. Leibovitch I, Mor Y. The vicious cycling: bicycling related urogenital disorders. Eur Urol. 2005;47:277-87. 